\title{
Review
}

\section{Gastric Diverticulum: A Comprehensive Review}

\author{
Jamil Shah $^{\mathrm{a}}$ Kalpesh Patel ${ }^{\mathrm{a}}$ Tagore Sunkara $^{\mathrm{b}}$ Charilaos Papafragkakis ${ }^{\mathrm{c}}$ \\ Abul Shahidullah ${ }^{d}$ \\ aDivision of Gastroenterology and Hepatology, Department of Medicine, Rutgers New Jersey Medical School, \\ University Hospital, Newark, NJ, USA; 'b Department of Gastroenterology, Mercy Medical Center, Des Moines, IA, USA; \\ 'Department of Gastroenterology, Metropolitan General Hospital, Athens, Greece; ${ }^{d}$ Department of Medicine, Henry \\ J. Carter Specialty Hospital and Nursing Facility, New York, NY, USA
}

\section{Keywords}

Gastric diverticulum · Stomach diverticulum · Diverticula

\begin{abstract}
Background: Gastric diverticula (GD) are outpouchings of the stomach wall that typically form in the fundus. They are the least common gastrointestinal diverticula, and are very rare anatomic abnormalities overall. Summary: Although most GD are asymptomatic and are usually discovered incidentally during routine diagnostic testing, they can present with variable symptoms. Occasionally, complications can develop that may be life-threatening and are indications for surgical management. Key Messages: Here, we provide a literature review of GD where we discuss the pathogenesis, clinical manifestations, investigations and diagnostic approach, and various management options for this uncommon disorder.

(c) 2019 S. Karger AG, Basel
\end{abstract}

\section{Introduction}

Gastric diverticula (GD) are outpouchings of the stomach wall that often form in the fundus, most frequently along the posterior wall [1]. They have features similar to those of small bowel diverticula and colonic diverticula [2]. GD are the least common gastrointestinal diverticula, and are very rare anatomic abnormalities overall. In fact, they are usually discovered incidentally during routine diagnostic testing. The rates of detection vary depending upon the method used to detect them. Estimates of prevalence range from $0.04 \%(165 / 380,000)$ in upper gastrointestinal contrast radiographic studies to $0.01-0.11 \%$ in upper gastrointestinal endoscopies to $0.02 \%(6 / 29,900)$ in autopsy studies $[1,3-5]$. In an early autopsy series, a prevalence of $0.1-2.6 \%$ was reported [6]. However, this wide range of prevalence has not been supported by the most recent literature, and GD are likely far less common than $2.6 \%$. They arise with equal distribution between men and women, and patients characteristically present between 50 and 70 years of age.

Although most individuals with GD are asymptomatic, others can present with variable abdominal symptoms, including epigastric pain and discomfort, nausea, vomiting, dyspepsia, early satiety, a vague sensation of postprandial fullness, belching, halitosis, anorexia, and dysphagia [5, 7-9]. Occasionally, complications, such as ulceration, upper gastrointestinal bleeding, hemorrhage, perforation, and malignant transformation, can develop that may be life-threatening and are indications for surgical management $[9,10]$. The rarity of this disorder, as well

\section{KARGER}

(c) 2019 S. Karger AG, Basel 
as the presence of a vague and nonspecific symptomatology, necessitates a high clinical index of suspicion from the internist, gastroenterologist, or surgeon.

A literature search on GD was performed using the PubMed search engine. The "keywords" above were used to locate relevant journal articles. Here, we provide a comprehensive literature review of this uncommon disorder.

\section{Pathogenesis}

GD can be classified into two types - congenital, or true, diverticula and acquired, or false, diverticula - with congenital diverticula being more common [3, 11-15]. True diverticula contain all layers of the gastric wall. In contrast, false diverticula do not. Furthermore, false diverticula can be classified into two types - pulsion and traction. Pulsion diverticula develop as a result of increased intraluminal pressure, such as from chronic coughing and from obesity. Conversely, traction diverticula develop as a result of contractile forces - either from an adjacent inflammatory process, or from perigastric adhesions from coexisting illnesses. These include peptic ulcer disease, pancreatitis, cholecystitis, cancer, gastric outlet obstruction, and gastroesophageal reflux disease $[9,16,17]$. In particular, traction diverticula in the stomach have been reported to occur following surgical procedures, including Roux-en-Y gastric bypass surgery $[6,17,18]$.

Although GD can develop nearly anywhere along the stomach wall, the two main types usually occur in different locations. Congenital diverticula, which compose 70$75 \%$ of all GD, are typically situated along the dorsal wall of the fundus, $2-3 \mathrm{~cm}$ below the gastroesophageal junction and $3 \mathrm{~cm}$ from the lesser curve [3]. Interestingly, congenital diverticula in the prepyloric area are likely to be associated with aberrant pancreatic tissue, a relatively frequent finding [13]. In contrast, acquired diverticula, also referred to as pseudodiverticula, are less frequent and are typically situated in or near the antrum [3]. GD are mostly solitary lesions that vary in size from 1 to $5 \mathrm{~cm}$ in diameter, with infrequent reports describing diverticula as large as $10-11 \mathrm{~cm}[16,19,20]$.

It is hypothesized that congenital diverticula develop due to the dividing of the longitudinal muscle fibers at the level of the cardia, leaving only the circular muscle fibers in the wall of the stomach and, as a result, forming a weak area through which a diverticulum can develop during the fetal period. This premise was supported by Reich
[21], who described GD in the fetus, as well as by Lewis [22], who reported gastrointestinal diverticula in the fetus as early as 1908.

\section{Clinical Manifestations}

Although most individuals with GD are asymptomatic, others can present with variable abdominal symptoms, including epigastric pain and discomfort, nausea, vomiting, dyspepsia, early satiety, a vague sensation of postprandial fullness, belching, halitosis, anorexia, and dysphagia [5, 7-9]. The most common symptom is upper abdominal pain, which has been reported in $18-30 \%$ of cases $[3,6]$. In fact, patients may report vague upper abdominal pain without any additional symptoms [7]. Other patients may report persistent, progressively worsening symptoms of localized epigastric pain, heartburn, and nausea despite proton pump inhibitor (PPI) therapy - complaints that are typical of severe gastroesophageal reflux disease [23-25]. Vomiting and dyspepsia are less common [6]. Still other patients may report a long history of frequent belching and a socially disabling halitosis, possibly due to retention of food and bacterial overgrowth inside the GD [5, 7, 8, 25].

It has been hypothesized that the size of the diverticular neck may be related to the development of symptoms. Often, GD with a wide neck are asymptomatic, possibly because food as well as digestive juices have a low tendency to become trapped. It has been hypothesized that retention of food followed by distention of the GD may produce pain $[26,27]$.

Infrequently, individuals with GD can develop complications, such as ulceration, upper gastrointestinal bleeding, hemorrhage, perforation, and malignant transformation, which may be life-threatening and are indications for surgical management $[9,10]$. It has been hypothesized that retention of food followed by the release of digestive juices inside the mucosal sac can cause inflammation, resulting in diverticulitis and possibly ulceration, hemorrhage, or even perforation [26, 27]. Also, GD have been reported very rarely to be associated with abscess formation and adenocarcinoma [28-31].

Since the clinical manifestations of GD can mimic those of other prevalent gastrointestinal illnesses, it can be a challenge to diagnose. Conversely, GD may only exacerbate complaints that are triggered by more prevalent gastrointestinal pathologies. Palmer [16] discovered that in 30 of 49 patients with GD who were symptomatic, the clinical manifestations could be attributed to other gastrointestinal disorders. 
Fig. 1. a, b Upper gastrointestinal contrast radiographic study. Oral contrast was administered through a gastrostomy tube. The patient was discovered to have a gastric diverticulum (arrows) along the greater curvature of the distal stomach.

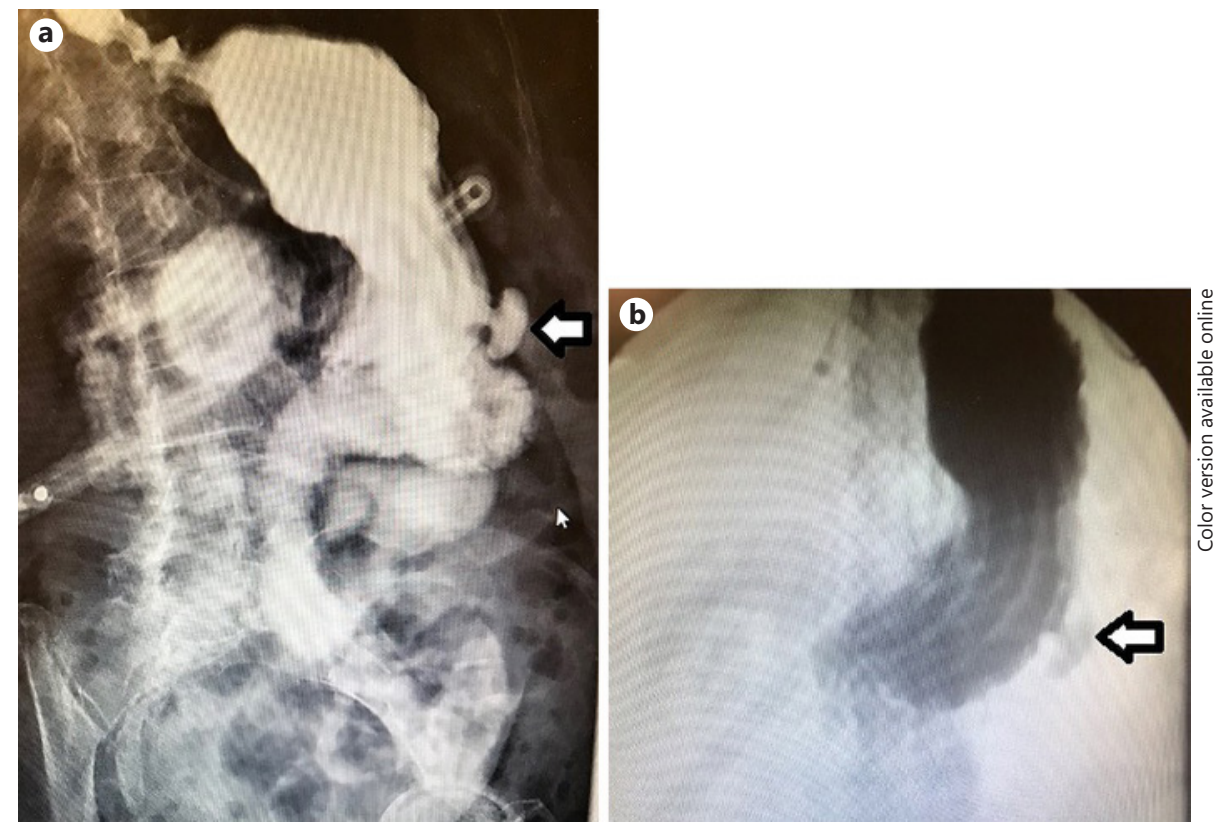

\section{Investigations}

In cases of GD, making an accurate diagnosis is very important given the possibility of uncommon, but severe, complications as well as the correlation with an ectopic mucosa and the potential for malignant conversion [2931]. The reports of gastric cancer found in and around GD are especially concerning. Thus, it is advised to perform a careful endoscopic examination and obtain biopsies from GD with suspicious, raised, or irregular borders. More often, GD are usually discovered incidentally during routine diagnostic investigation of common gastrointestinal symptoms. However, the methods used to detect them can fail, and, thus, a combined diagnostic approach should be used [32]. GD can be identified through radiological examinations (upper gastrointestinal contrast radiographic study and abdominal CT scanning with oral contrast administration) and upper gastrointestinal endoscopic examinations (Fig. 1-3).

Upper gastrointestinal contrast radiographic studies (UGI series) and esophagogastroduodenoscopy (EGD) are the most reliable diagnostic methods to verify the presence of a diverticulum. However, UGI series occasionally fail to establish the diagnosis, mainly due to nonopacification with contrast of the diverticular sac due to a narrow diverticular neck $[3,16,20]$. Indeed, in a large review, Palmer [16] determined that 14 of 262 GD (5\%) failed to be observed during UGI studies. It has been shown that during UGI series, the GD is best recognized with the use of a right, anterior oblique angle while the individual is in a Trendelenburg and slightly left lateral decubitus position $[16,20,33,34]$. It is imperative to obtain the lateral view in order to detect the outpouching of the diverticulum along the dorsal wall of the stomach [35].

By comparison, reports in the literature advise using EGD, the gold standard examination, to make an accurate diagnosis $[18,36]$. This modality is useful not only in confirming the GD's location and size, but also in providing the opportunity to perform a biopsy if a coexisting illness is suspected.

In general, the use of CT scans as part of the diagnostic workup for individuals with GD is not widely accepted due to potential misdiagnoses. There are many reports in the literature of cases that were misdiagnosed as left adrenal masses by CT imaging, with extensive unnecessary endocrinology workup and even exploratory laparotomy conducted in some cases [37-40]. In some of these misreads of $\mathrm{CT}$, the radiologist failed to identify an air bubble in the lesion, which signifies continuation with the gastric lumen and not a left adrenal mass. Given that most GD are situated along the dorsal wall of the stomach, it has been suggested that CT scans conducted in the prone position may assist in making the correct diagnosis, as this position may facilitate air movement to the top and formation of an air-fluid level [41]. 
Fig. 2. a, b Endoscopic view of a gastric diverticulum (white arrows) located in the antrum. The pylorus (black arrows) is visible in the lower left part of the images.

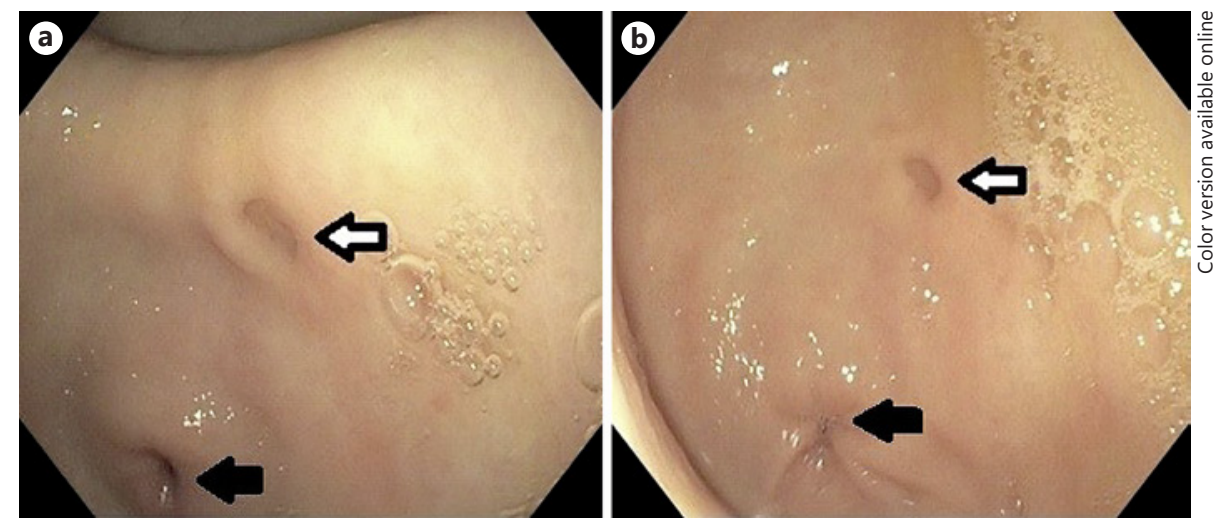

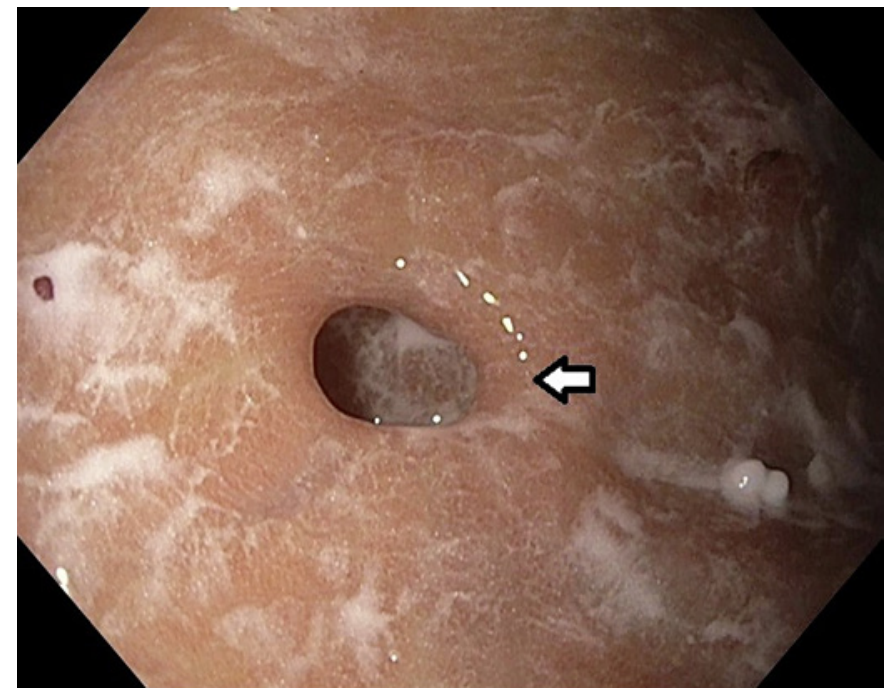

Fig. 3. Endoscopic view of a gastric diverticulum (white arrow) located in the body of the stomach.

Conversely, some reports suggest that CT scans with oral contrast may, in fact, be useful in diagnosing GD, particularly when MRI has failed [25, 41-43]. Thus, although most previously reported cases have utilized UGI series to confirm the diagnosis, these cases suggest that CT imaging, specifically when enhanced with oral contrast, may assist in differentiating GD from adrenal masses. Furthermore, in yet another case of a GD imitating an adrenal adenoma on imaging, examining both the axial and coronal sections of an abdominal CT scan was found to be useful in differentiating a left adrenal gland lesion from a non-adrenal gland lesion such as a GD [43].

\section{Management}

The management of GD depends largely on the severity of the presenting complaints as well as on the size of the diverticulum. It is well documented that asymptomatic individuals do not require any treatment and can be left alone [16, 21, 44, 45]. The majority of diverticula is congenital, accidentally discovered, and asymptomatic, and therefore requires no treatment. Diverticula associated with significant symptoms or leading to complications - these diverticula are usually large in size - should be resected as there is no other effective treatment.

\section{Medical and Nonsurgical Management}

Patients with GD who are symptomatic should initially be treated with PPI therapy, histamine $\mathrm{H}_{2}$ receptor antagonist therapy, or antacid therapy. PPI therapy for several weeks has been reported to alleviate symptoms in confirmed cases of GD [44]. Some authors support conservative treatment with antacid therapy, but this is with the awareness that symptom relief may be temporary [3, 9]. In fact, several studies describe patients who presented soon after with refractory symptoms of worsening abdominal pain and dyspepsia that failed to improve with either PPIs, $\mathrm{H}_{2}$ antagonists, or antacids [23, 46]. GD greater than $4 \mathrm{~cm}$ in diameter are more susceptible to complications and are less likely to respond favorably to medical management [9]. Patients who do not respond to the aforementioned treatment should undergo a diagnostic EGD or UGI series.

Several reports have described successful endoscopic management of patients with GD who presented with acute upper gastrointestinal bleeding [2,47]. No studies reported further complications that called for surgical management. Thus, EGD has an essential role in the 
management of acute upper gastrointestinal bleeding due to GD.

In 1 case, endoscopic resection was applied to an abnormally shaped, inverted-type, early gastric cancer (EGC), which was shaped similar to a GD as a result of submucosal inflammation and infiltration [31]. The lesion was successfully treated with endoscopic submucosal dissection, an endoscopic maneuver that has been established as a standard treatment option for EGC that does not carry the possibility of lymph node or distant metastases. This case is a unique report of an endoscopic submucosal dissection performed on an EGC that happened to be associated with a small diverticulum. It was resected successfully without complications.

\section{Surgical Management}

Surgical resection continues to be the main treatment when the GD are large ( $>4 \mathrm{~cm}$ in diameter), patients are still symptomatic after PPI therapy, and complications, such as ulceration, upper gastrointestinal bleeding, hemorrhage, perforation, and malignant transformation, have occurred. Palmer $[16,45]$ reported that two-thirds (6 of 9 ) of symptomatic patients with GD who underwent surgical resection had excellent outcomes and remained symptom free after the surgery.

Both open and laparoscopic surgical resections lead to excellent results, although, currently, the laparoscopic approach is preferred. In 2 cases, described earlier in this literature review, this approach was effectively performed without any technical difficulties $[8,25]$. In experienced hands, a laparoscopic dissection is frequently easier to perform. By comparison, the minimally invasive surgical approach can occasionally be challenging because GD are frequently collapsed, concealed in the splenic bed, or deeply adherent to the posterior surface of the pancreas $[16,46]$. In short, laparoscopic resection is both a safe and feasible surgical approach with excellent outcomes, and is indicated for individuals with GD who are symptomatic.

Resection of the incorrect part of the stomach has been reported, as well [18]. Therefore, it should be strongly considered to combine the surgical procedure with intra- operative endoscopy. This would allow for the discovery of elusive GD by stretching the diverticular sac $[5,48-50]$, especially as reoperation for posterior GD has been reported [51].

\section{Conclusions}

GD are rare and require a high clinical index of suspicion by the physician. Vague symptoms, such as pain, discomfort, and dyspepsia, may be related to their presence. Other hygiene-related symptoms with a serious social impact, such as halitosis, may trigger specific investigation for GD. UGI series and EGD play important roles in the diagnosis of GD. Laparoscopic resection, sometimes assisted by intraoperative endoscopy, is a safe and feasible surgical treatment that should be offered when GD are large, patients have not responded adequately to medical treatment, and complications have occurred.

\section{Statement of Ethics}

The authors have no ethical conflicts to disclose.

\section{Disclosure Statement}

The authors have no conflicts of interest to declare.

\section{Funding Sources}

The authors declare that neither financial nor grant support has been received for the preparation of this manuscript.

\section{Author Contributions}

T.S. conceived the idea for the review. J.S. designed and drafted the review. K.P., T.S., C.P., and A.S. evaluated and revised the manuscript critically for important intellectual content. K.P. and A.S. provided the images.

\section{References}

Gastric Diverticulum: A Comprehensive

Review
1 Gockel I, Thomschke D, Lorenz D. Gastrointestinal: gastric diverticula. J Gastroenterol Hepatol. 2004 Feb;19(2):227.

2 Chen JH, Su WC, Chang CY, Lin H. Education and imaging. Gastrointestinal: bleeding gastric diverticulum. J Gastroenterol Hepatol. 2008 Feb;23(2):336.
3 Rodeberg DA, Zaheer S, Moir CR, Ishitani MB. Gastric diverticulum: a series of four pediatric patients. J Pediatr Gastroenterol Nutr. 2002 May;34(5):564-7.

4 Schiller AH, Roggendorf B, Delker-Wegener S, Richter K, Kuthe A. [Laparoscopic resection of gastric diverticula: two case reports]. Zentralbl Chir. 2007 Jun;132(3):251-5. 
5 Donkervoort SC, Baak LC, Blaauwgeers JL, Gerhards MF. Laparoscopic resection of a symptomatic gastric diverticulum: a minimally invasive solution. JSLS. 2006 Oct-Dec; 10(4):525-7.

6 Meeroff M, Gollán JR, Meeroff JC. Gastric diverticulum. Am J Gastroenterol. 1967 Mar; 47(3):189-203.

7 Podda M, Atzeni J, Messina Campanella A, Saba A, Pisanu A. Syncope with surprise: an unexpected finding of huge gastric diverticulum. Case Rep Surg. 2016;2016:1941293.

8 Muis MO, Leitao K, Havnen J, Glomsaker TB, Søreide JA. Gastric diverticulum and halitosis-A case for surgery? Int J Surg Case Rep. 2014;5(7):431-3.

9 DuBois B, Powell B, Voeller G. Gastric diverticulum: "a wayside house of ill fame" with a laparoscopic solution. JSLS. 2012 Jul-Sep; 16(3):473-7.

10 Ho MF, Teoh AY, Liu SY, Chiu PW, Wong SK, Ng EK. Perforated gastric diverticulum with bleeding. Endoscopy. 2013;45 Suppl 2 UCTN:E422-3.

11 Akerlund D. Gastric diverticulum. Acta Radiol. 1923;2:476-85.

12 Schmidt HW, Walters W. Diverticula of stomach. Surg Gynecol Obstet. 1935;60:106.

13 Wolters VM, Nikkels PG, Van Der Zee DC, Kramer PP, De Schryver JE, Reijnen IG, et al. A gastric diverticulum containing pancreatic tissue and presenting as congenital double pylorus: case report and review of the literature. J Pediatr Gastroenterol Nutr. 2001 Jul;33(1): 89-91.

14 Cotea E, Vasilescu A, Dimofte G, Crumpei F, Moldovanu R, Mihăilescu A. Gastric diverticula on the greater curvature. J Chir Iasi. 2007;3:269-73.

15 Love L, Meyers MA, Churchill RJ, Reynes CJ, Moncada R, Gibson D. Computed tomography of extraperitoneal spaces. AJR Am J Roentgenol. 1981 Apr;136(4):781-9.

16 Palmer ED. Gastric diverticula. Int Abstr Surg. 1951 May;92(5):417-28.

17 Schweiger F, Noonan JS. An unusual case of gastric diverticulosis. Am J Gastroenterol. 1991 Dec;86(12):1817-9.

18 Anaise D, Brand DL, Smith NL, Soroff HS Pitfalls in the diagnosis and treatment of a symptomatic gastric diverticulum. Gastrointest Endosc. 1984 Feb;30(1):28-30.

19 Palmer ED. Gastric diverticula, with special reference to subjective manifestations. Gastroenterology. 1958 Oct;35(4):406-8.

20 Seltzer MH, Koch AW. A huge gastric diverticulum. Am J Dig Dis. 1971 Feb;16(2):167-70.
21 Reich NE. Gastric diverticula. Am J Dig Dis. 1941;8(3):70-6.

22 Lewis FT, Thyng FW. Regular occurrence of intestinal diverticula in embryos of pig, rabbit and man. Am J Anat. 1908;7(4):505-19.

23 Moy BT, Marchioni Beery RM, Birk JW. Gastric diverticulum: an unusual endoscopic finding. ACG Case Rep J. 2016 Apr;3(3):1501.

24 Hajini FF, Husain M, Bhat A, Bukhari SI. Gastric diverticulum a rare endoscopic finding. BMJCase Rep.2014Apr;2014:bcr2013202887.

25 Globke B, Fikatas P, Beck A, Adler A, Schmidt SC. Multidisciplinary laparo-endoscopic management of a subcardial gastric diverticulum. Endoscopy. 2013;45 Suppl 2 UCTN: E138-9.

26 Kilkenny JW 3rd. Gastric diverticula: it's time for an updated review. Gastroenterology. 1995 Apr;108(4):A1226.

27 Tillander H, Hesselsjö R. Juxtacardial gastric diverticula and their surgery. Acta Chir Scand. 1968;134(3):255-63.

28 Pietrzak C, Leya J, Abood G, Yonick D, Gupta N. Recurrent intra-abdominal abscess formation due to a gastric diverticulum. Endoscopy. 2013;45 Suppl 2 UCTN:E345-6.

29 Fork FT, Tóth E, Lindström C. Early gastric cancer in a fundic diverticulum. Endoscopy. 1998 Jan;30(1):S2.

30 Adachi Y, Mori M, Haraguchi Y, Sugimachi K. Gastric diverticulum invaded by gastric adenocarcinoma. Am J Gastroenterol. 1987 Aug;82(8):807.

31 Lee YI, Lee SK. Endoscopic submucosal dissection of an inverted early gastric cancerforming false gastric diverticulum. Clin Endosc. 2016 Jan;49(1):86-90.

32 Simon M, Zuber-Jerger I, Schölmerich J. True gastric diverticulum. Dig Liver Dis. 2009 May; 41(5):370.

33 Bothén NF, Eklöf O. Diverticula and duplications (enterogenous cysts) of the stomach and duodenum. Am J Roentgenol Radium Ther Nucl Med. 1966 Feb;96(2):375-81.

34 Eras P, Beranbaum SL. Gastric diverticula: congenital and acquired. Am J Gastroenterol. 1972 Feb;57(2):120-32.

35 Eastwood SR. Diverticulum of the stomach. Proc R Soc Med. 1929 Jul;22(9):1234-5.

36 Velanovich V. Gastric diverticulum. Endoscopic and radiologic appearance. Surg Endosc. 1994 Nov;8(11):1338-9.

37 Nakajo M, Umanodan T, Nakajo M. A case of bilateral adrenal pseudotumors caused by hepatic cavernous hemangioma and gastric diverticulum. Jpn J Clin Radiol. 2002;47:573-7.
38 Kodera R, Otsuka F, Inagaki K, Miyoshi T, Ogura T, Tanimoto Y, et al. Gastric diverticulum simulating left adrenal incidentaloma in a hypertensive patient. Endocr J. 2007 Dec; 54(6):969-74.

39 Araki A, Shinohara M, Yamakawa J, Tanaka M, Natsui S, Izumi Y. Gastric diverticulum preoperatively diagnosed as one of two left adrenal adenomas. Int J Urol. 2006 Jan;13(1): 64-6.

40 Schwartz AN, Goiney RC, Graney DO. Gastric diverticulum simulating an adrenal mass: CT appearance and embryogenesis. AJR Am J Roentgenol. 1986 Mar;146(3):553-4.

41 Feng YE, Zhang Z. Gastric diverticulum simulating a left adrenal mass: a case report and review of the literature. Oncol Lett. 2015 Oct; 10(4):2477-80.

42 Mahafza WS, Taib AA, Shahait AD, Al Awamleh A. Chronic gastritis in a gastric diverticulum misdiagnosed as a left adrenal mass. Indian J Surg. 2015 Apr;77(S1 Suppl 1): 150-2.

43 Jebasingh FK, Naik D, Chandramohan A, Paul MJ. Posterior gastric diverticulum mimicking adrenal adenoma on imaging. BMJ Case Rep. 2014 Dec;2014:bcr2014207059.

44 Mohan P, Ananthavadivelu M, Venkataraman J. Gastric diverticulum. CMAJ. 2010 Mar;182(5):E226.

45 Palmer ED. Gastric diverticulosis. Am Fam Physician. 1973 Mar;7(3):114-7.

46 MaCauley M, Bollard E. Gastric diverticulum: a rare cause of refractory epigastric pain. Am J Med. 2010 May;123(5):e5-6.

47 Zakary NY, van Langenberg DR, Alshumrany M, Schoeman MN. Gastrointestinal: acute haemorrhage from a Dieulafoy lesion within a gastric diverticulum managed endoscopically. J Gastroenterol Hepatol. 2009 Dec; 24(12):1891.

48 Fine A. Laparoscopic resection of a large proximal gastric diverticulum. Gastrointest Endosc. 1998 Jul;48(1):93-5.

49 Vogt DM, Curet MJ, Zucker KA. Laparoscopic management of gastric diverticula. J Laparoendosc Adv Surg Tech A. 1999 Oct;9(5): 405-10.

50 Kim SH, Lee SW, Choi WJ, Choi IS, Kim SJ, Koo BH. Laparoscopic resection of gastric diverticulum. J Laparoendosc Adv Surg Tech A. 1999 Feb;9(1):87-91.

51 Smith RS, Mortensen JD. Diverticulum of the gastric cardia. Northwest Med. 1949 Mar; 48(3):185-8. 